\title{
Refined prediction: Does improved accuracy enhance our ability to improve patient outcomes?
}

\author{
Bryan A. Whitson, MD, PhD
}

\author{
From the Division of Cardiac Surgery, Department of Surgery, The Ohio State University Medical Center, \\ Columbus, Ohio. \\ Disclosures: Author has nothing to disclose with regard to commercial support. \\ Received for publication March 12, 2016; accepted for publication March 14, 2016. \\ Address for reprints: Bryan A. Whitson, MD, PhD, Division of Cardiac Surgery, COPPER Laboratory \\ Department of Surgery, The Ohio State University Medical Center, N-816 Doan Hall, 410 W 10th Ave, \\ Columbus, OH 43210 (E-mail: bryan.whitson@osumc.edu). \\ J Thorac Cardiovasc Surg 2016;152:147 \\ $0022-5223 / \$ 36.00$ \\ Copyright (c) 2016 by The American Association for Thoracic Surgery \\ http://dx.doi.org/10.1016/j.jtcvs.2016.03.036
}

Acute kidney injury (AKI) is unfortunately all too prevalent after cardiac surgery, with reported incidences of up to $24 \%$ to $30 \%{ }^{1,2}$ with approximately $1 \%$ of patients requiring dialysis, often permanently. ${ }^{1}$ Furthermore, patients who develop AKI have an time, our ability to measure renal insufficiency has been reflected in the glomerular filtration rate and the serum creatinine, which may be influenced by lean body mass, activity, and diet, as examples.

Dardashti and colleagues ${ }^{3}$ present their work investigating the utility of an alternative marker of AKI, (s)cystatin $\mathrm{C}$, in the prediction of mortality after coronary artery bypass grafting (CABG) surgery. In their analysis, Dardashti and colleagues ${ }^{3}$ examine a single institution dataset of 1638 patients who underwent CABG. The patients had serum (s)-cystatin C levels measured and were followed for a median of 3.5 years (range, 2-5 years) to measure outcomes and mortality. In that cohort, the 30-day mortality was a very respectable $0.8 \%$. The authors are to be commended on their excellent outcomes and their comprehensive follow-up over time that was no doubt a result of their ability to follow an integrated (health and tax) system.

In this analysis, (s)-cystatin $\mathrm{C}$, a low-molecular-weight protein byproduct that is filtered by the glomeruli and is elevated in the serum with decreased filtration, was measured perioperatively in addition to serum creatinine level and estimated glomerular filtration rate. Dardashti and colleagues ${ }^{3}$ demonstrate that (s)-cystatin C leveland the estimated glomerular filtration rate based on that measurement-is a precise predictor of 30-day and long-term mortality. Although (s)-cystatin $\mathrm{C}$ has some shortcomings (on which the authors comment), it was a more accurate predictor of mortality when measured pre-CABG and during the perioperative period. In their receiver operating curve analysis, a preoperative (s)-cystatin C cutoff value of 0.9 demonstrated $96.4 \%$ sensitivity and $79 \%$ specificity in discriminatory accuracy for mortality. Although controversial, ${ }^{4}$ if (s)-cystatin $\mathrm{C}$ is indeed a marker of renal dysfunction that has a high

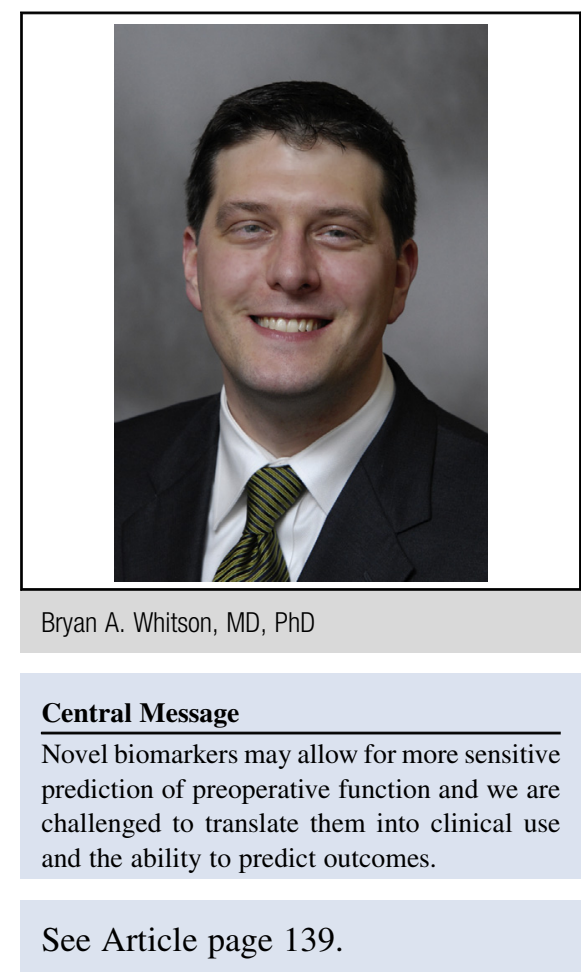

accuracy of predicting mortality, are we able to use that information to implement known renal-protection measures before, during, and after surgery? ${ }^{1}$ (s)-Cystatin $\mathrm{C}$ is an evolving biomarker and tool for measuring AKI that appears to be more sensitive than s-creatinine. (s)-Cystatin $\mathrm{C}$ appears to be better either alone or in combination with (s)-creatinine. (s)-Cystatin $\mathrm{C}$ would appear to be less influenced by lean body mass and other factors than creatinine. If these data can be translated into a reproducible, cost-effective metric that allows for active intervention to correct or prevent $\mathrm{AKI}$, then preoperative (s)-cystatin $\mathrm{C}$ measurement becomes a powerful clinical tool.

\section{References}

1. Rosner MH, Okusa MD. Acute kidney injury associated with cardiac surgery. Clin J Am Soc Nephrol. 2006;1:19-32.

2. Karkouti K, Wijeysundera DN, Yau TM, Callum JL, Cheng DC, Crowther M, et al Acute kidney injury after cardiac surgery: focus on modifiable risk factors. Circulation. 2009;119:495-502.

3. Dardashti A, Nozohoor S, Algotsson L, Ederoth P, Bjursten H. The predictive value of cystatin $\mathrm{C} 1$ for mortality after coronary artery bypass surgery. J Thorac Cardiovasc Surg. 2016;152:139-46.

4. Hojs R, Bevc S, Ekart R, Gorenjak M, Puklavec L. Serum cystatin C-based equation compared to serum creatinine-based equations for estimation of glomerular filtration rate in patients with chronic kidney disease. Clin Nephrol. 2008;70:10-7. 